\title{
openheart Prevalence of left ventricle non- compaction criteria in adult patients with bicuspid aortic valve versus healthy control subjects
}

\author{
Mylène Shen, ${ }^{1}$ Romain Capoulade, ${ }^{1}$ Lionel Tastet, ${ }^{1}$ Ezequiel Guzzetti, ${ }^{1}$ \\ Marie-Annick Clavel, ${ }^{1}$ Erwan Salaun, ${ }^{1}$ Élisabeth Bédard, ${ }^{1}$ Marie Arsenault, ${ }^{1}$ \\ Philippe Chetaille, ${ }^{2}$ Helena Tizón-Marcos, ${ }^{1}$ Florent Le Ven, ${ }^{1}$ Philippe Pibarot, ${ }^{1}$ \\ Éric Larose ${ }^{1}$
}

\begin{abstract}
- Additional material is published online only. To view please visit the journal online (http://dx.doi.org/10.1136/ openhrt-2018-000869)
\end{abstract}

To cite: Shen M, Capoulade R, Tastet $L$, et al. Prevalence of left ventricle non-compaction criteria in adult patients with bicuspid aortic valve versus healthy control subjects. Open Heart 2018;5:e000869. doi:10.1136/ openhrt-2018-000869

Received 30 May 2018 Revised 8 August 2018 Accepted 14 August 2018

Check for updates

(c) Author(s) (or their employer(s)) 2018. Re-use permitted under CC BY-NC. No commercial re-use. See rights and permissions. Published by BMJ.

${ }^{1}$ Institut Universitaire de Cardiologie et de Pneumologie de Québec / Quebec Heart \& Lung Institute, Laval University, Quebec City, Quebec, Canada ${ }^{2}$ Department of Pediatrics, Centre Mère Enfants Soleil, Centre Hospitalier Universitaire de Québec, Laval University, Quebec City, Quebec, Canada

Correspondence to Dr Philippe Pibarot; philippe. pibarot@med.ulaval.ca

\section{ABSTRACT}

Objective The aim of this study was to compare the prevalence of left ventricle non-compaction (LVNC) criteria (or hypertrabeculation) in a cohort of patients with bicuspid aortic valve (BAV) and healthy control subjects (CTL) without cardiovascular disease using cardiovascular MR (CMR).

Methods 79 patients with BAV and 85 CTL with tricuspid aortic valve and free of known cardiovascular disease underwent CMR to evaluate the presence of LVNC criteria. The left ventricle was assessed at end-systole and enddiastole, in the short-axis, two-chamber and four-chamber views and divided into the 16 standardised myocardial segments. LVNC was assessed using the non-compacted/ compacted (NC/C) myocardium ratio and was considered to be present if at least one of the myocardial segments had a NC/C ratio superior to the cut-off values defined in previous studies: Jenni et al (>2.0 end-systole); Petersen et al (>2.3 end-diastole); or Fazio et al (>2.5 end-diastole). Results 15 CTL (17.6\%) vs 8 BAV (10.1\%) fulfilled Jenni et af's criterion; 69 CTL $(81.2 \%)$ vs 49 BAV $(62.0 \%)$ fulfilled Petersen et a/s criterion; and 66 CTL (77.6\%) vs 43 BAV (54.4\%) fulfilled Fazio et al's criterion. Petersen et al and Fazio et a/s LVNC criteria were met more often by CTL ( $p=0.006$ and $p=0.002$, respectively) than patients with $B A V$, whereas this difference was not statistically significant according to Jenni et a/s criterion ( $\mathrm{p}=0.17)$. In multivariable analyses, after adjusting for age, sex, the presence of significant valve dysfunction (>mild stenosis or >mild regurgitation), indexed LV mass, indexed LV enddiastolic volume and LV ejection fraction, BAV was not associated with any of the three LVNC criteria.

Conclusion Patients with BAV do not harbour more LVNC than the general population and there is no evidence that they are at higher risk for the development of LVNC cardiomyopathy.

\section{INTRODUCTION}

A myocardium with left ventricle non-compaction (LVNC) or hypertrabeculation typically presents a thin, dense and compacted epicardial layer and a thick, spongious and

\section{Key questions}

What is already known about this subject?

- Bicuspid aortic valve (BAV), the most common congenital heart disease, is often associated with other types of congenital anomalies or syndromes. Some previous studies have suggested that patients with BAV may have a higher prevalence of left ventricle non-compaction (LVNC) than the general population.

What does this study add?

- This study compared the prevalence of LVNC criteria in patients with BAV versus healthy control subjects by using cardiovascular MR (CMR) and three previously published methods. Patients with BAV did not meet the LVNC criteria more often than healthy subjects.

How might this impact on clinical practice?

- There is no evidence that the patients with BAV might be at higher risk to develop LVNC cardiomyopathy and systematic screening for LVNC by CMR does not appear necessary in subjects with BAV.

non-compacted endocardial layer. ${ }^{1}$ LVNC is a rare congenital cardiomyopathy characterised by prominent trabeculations and deep intertrabecular recesses in the left ventricle (LV). LVNC is believed to be the result of an early arrest of the compacting processes of the myocardium during embryonic life, although rare cases of acquired LVNC have been described. $^{2-5}$ The exact prevalence of LVNC in the population is unknown but according to previous echocardiographic studies, it may vary from $0.05 \%$ to $1.26 \%$ in general ${ }^{6-9}$ and may reach $3 \%-4 \%$ in patients with heart failure or decreased left ventricle ejection fraction (LVEF). ${ }^{10}$ The clinical presentation of LVNC is heterogeneous, ranging from an absence of symptoms to very serious complications including ventricular arrhythmias, 
heart failure or systemic embolic events. ${ }^{24}$ The American Heart Association (AHA)/American College of Cardiology classify LVNC as a genetic cardiomyopathy but the European Society of Cardiology and the World Health Organization consider this entity as an unclassified cardiomyopathy. ${ }^{11-13}$ Furthermore, it remains controversial whether LVNC is a distinct cardiomyopathy or a common feature shared by several cardiomyopathies. ${ }^{5}$

Bicuspid aortic valve (BAV) is the most common congenital heart disease in the general population $(1 \%-2 \%)$ and has a higher prevalence in men versus women (ratio 3:1). ${ }^{14} 15$ This anomaly, characterised by a bileaflet aortic valve instead of a normal trileaflet aortic valve, is more prone to develop valve dysfunctions such as aortic stenosis (AS) or aortic regurgitation (AR). ${ }^{16}$ Subjects with BAV are also known for presenting other congenital anomalies and syndromes such as aortic coarctation, aortic aneurysms or Turner's syndrome. ${ }^{17} 18$ Due to its association with several other anomalies and syndromes, it is plausible that BAV subjects may harbour a higher prevalence of LVNC/hypertrabeculation and ensuing cardiomyopathy than the general population. To this effect, some previous echocardiographic studies have suggested that LVNC is more prevalent in patients with BAV than in the general population. ${ }^{19}{ }^{20}$ However, those studies used echocardiography to identify LVNC and they did not include a control group of healthy subjects with a morphologically normal aortic valve (tricuspid aortic valve) for comparison with the BAV subjects. Cardiovascular MR (CMR) allows a better characterisation of the LV morphology and function and a better delineation of the trabeculations than echocardiography. ${ }^{21} 22$

Thus, the aim of our study was to compare the prevalence of LVNC criteria in a cohort of patients with BAV and healthy control subjects (CTL) without cardiovascular disease using CMR.

\section{METHODS}

\section{Population of the study}

The population of the study consisted of 164 adults, 79 patients with BAV and 85 healthy CTL with tricuspid aortic valve. Patients with BAV who underwent a CMR exam between 21 November 2006 and 1 June 2015 at the Quebec Heart and Lung Institute were included in this study. They were either participants of the Metabolic Determinants of the Progression of Aortic Stenosis study (ClinicalTrials.gov NCT01679431) $(\mathrm{n}=40)$ or patients who were followed at the adult congenital heart diseases clinic $(n=39)$. The latter had CMR as part of their clinical follow-up, generally to assess the function of the aortic valve, the left ventricular function and volumes, the aorta dimensions, or to monitor the evolution of previous procedures (ie, repair of a coarctation of the aorta, surgery for anomalous pulmonary venous return, valvuloplasty, ventricular septal defect closure, etc). The CMR images of these patients were analysed retrospectively. BAV morphology was confirmed by CMR and/or
Doppler echocardiography. The control group consisted of subjects aged 18-35 years and free of any congenital or acquired cardiovascular disease, hypertension, dyslipidaemia, diabetes or renal, hepatic or blood disorders that were prospectively recruited in a previous study, which aimed at establishing normal reference values for $\mathrm{CMR} .{ }^{23}$ LVNC criteria were assessed in a subset of the subjects included in this previous CMR study. ${ }^{24}$ The study was approved by the institution ethics board and informed consent was obtained for subjects who were recruited prospectively and consent was waived for the BAV subjects who were assessed retrospectively.

\section{Cardiovascular MR}

All CTL and patients with BAV underwent CMR with a 1.5 T Philips Achieva scanner (release 2.6, level 3; Philips Healthcare, Best, The Netherlands) using the same imaging protocol. ${ }^{24}$ Briefly, cine imaging of cardiac morphology and function was performed by steady-state free precession technique at 30 phases per cardiac cycle in apnoea. Short-axis and three radial long-axis planes were performed covering the entire cardiac silhouette. Images were analysed off line in an experienced core laboratory using a standardised approach by trained technicians and supervised by an experienced cardiologist (EL). The analysis followed the 16-segment myocardial model ${ }^{25}$ and was performed using CMR Mass (version 7.1; Medis, Leiden, The Netherlands). Cardiac volumes and function measurements were performed as previously described. ${ }^{23} \mathrm{Left}$ ventricle end-diastolic volume (LVEDV) and end-systolic volume (LVESV), left ventricle stroke volume, LVEF and left ventricle mass (LVM) were computed using Simpson's rule adjusted to body surface area (BSA). Segmental wall thickness was measured at end-diastole by the centre line method and was compared with the average chord thickening at end-systole in each segment to determine the segmental wall function.

\section{Identification of LVNC by CMR}

LV trabeculated and non-trabeculated myocardium was specifically analysed by an experienced reader (EL). Slices from short-axis, two-chamber and four-chamber views were analysed in end-diastole and end-systole, in three short-axis planes (basal, mid and apical LV) and in a single mid-cavity plane of two-chamber and four-chamber views. Segmentation of the myocardium followed the AHA recommendations. LVNC was assessed by dividing the non-compacted (NC) myocardium thickness by the compacted (C) myocardium thickness for each of the 16 myocardial segments, thus providing $\mathrm{NC} / \mathrm{C}$ myocardium ratios (figure 1). Detailed methodology follows that of Tizón-Marcos et al. ${ }^{24}$

LVNC was considered to be present if at least one of the myocardial segments had a $\mathrm{NC} / \mathrm{C}$ ratio superior to the cut-off values defined in the following previous studies: Jenni et al: $>2.0$ at end-systole ${ }^{1}$; Petersen et al: $>2.3$ at end-diastole ${ }^{26}$; Fazio et al: $>2.5$ at end-diastole. ${ }^{27}$ The NC/C ratios for each myocardial segment, for each 


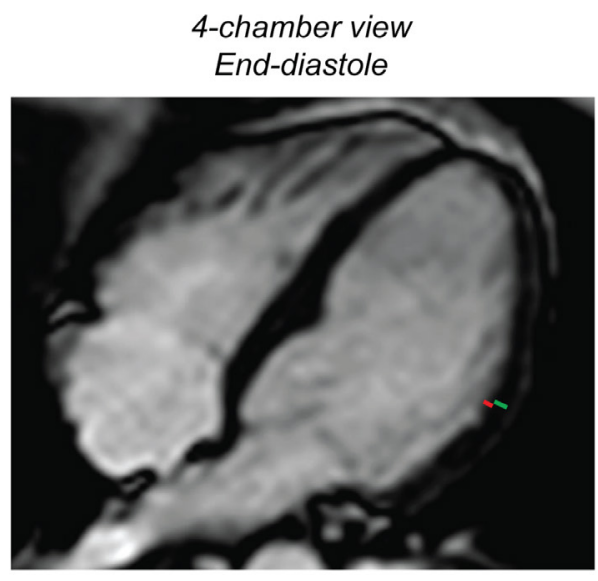

\section{LVNC criteria NOT fulfilled $\mathrm{NC} / \mathrm{C}$ ratio $=0.71$}

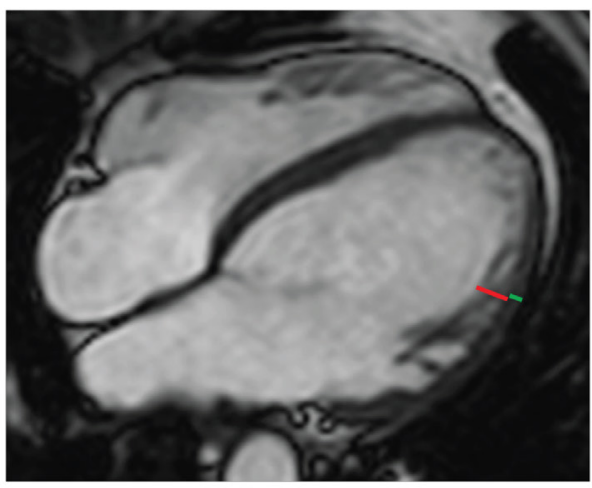

\section{LVNC criteria fulfilled $\mathrm{NC} / \mathrm{C}$ ratio $=\mathbf{2 . 5 4}$}

Non-compacted/trabeculated myocardium thickness

Figure 1 Patients without (top) and with (bottom) LVNC/hypertrabeculation criteria. Top: patient not fulfilling LVNC criteria. Bottom: patient fulfilling LVNC criteria. LVNC, left ventricle non-compaction; NC/C, non-compacted/compacted myocardium.

group, are presented in online supplementary tables 1-6. Intraobserver reproducibility for the measurements of NC myocardium thicknesses and (NC+C) myocardium thicknesses was excellent with $\rho=0.91$ and 0.97 , respectively.

\section{Statistical analyses}

The continuous variables were tested for normality of distribution and homogeneity of variances with the Shapiro-Wilk and Levene's tests, respectively. Continuous data with a normal distribution were expressed as mean $\pm \mathrm{SD}$, while continuous data with non-normal distribution were expressed as median (25th percentile-75th percentile). Comparisons between patients with BAV and CTL were done with Student's t-test or Wilcoxon-Mann-Whitney test as appropriate. Comparisons among patients with BAV (BAV without significant valve dysfunction (group ND), BAV with a predominant and significant (>mild) AS (group AS) and BAV with a predominant and significant (>mild) AR (group AR)) were done with analysis of variance or Kruskal-Wallis tests as appropriate. Categorical data were expressed as percentage and compared with the $\mathrm{X}^{2}$ test or Fisher's exact test as appropriate. Univariable and multivariable logistic regression analyses were used to assess the association between the BAV morphology and the three NC/C ratios diagnosing LVNC. Statistical analyses were performed with SPSS (V.23, SPSS) and JMP (V.12.1.0; SAS Institute, Cary, North Carolina) software programs and a $\mathrm{p}$ value $<0.05$ was considered statistically significant.

\section{RESULTS}

\section{Characteristics of the CTL and patients with BAV}

The demographic and CMR characteristics of the patients with BAV and CTL are presented in table 1. Patients with BAV were older (38 (29-51) vs 28 (24-31) years, $\mathrm{p}<0.0001)$, more often men ( $66 \%$ vs $44 \%$, $\mathrm{p}=0.004)$ and had larger body weight (73.1 (64.3-84.0) vs 67.1 (57.5-80.6) kg, p=0.046), body mass index (25.4 (22.827.7) vs $\left.23.5(21.3-26.4) \mathrm{kg} / \mathrm{m}^{2}, \mathrm{p}=0.01\right)$, LVEDV (163.0 (133.3-204.2) vs $144.5(127.8-168.7) \mathrm{mL}, \mathrm{p}=0.03)$ and LVESV (53.0 (40.7-80.7) vs 47.4 (38.4-56.3) mL, $\mathrm{p}=0.03)$ than CTL. The difference between BAV and CTL with respect to $\mathrm{LV}$ volumes persisted when indexed to BSA $(p=0.07$ and $p=0.02$, respectively, for indexed LVEDV (LVEDVi) and indexed LVESV (LVESVi)). Furthermore, LVM was higher in patients with BAV than in CTL (131.5 (97.6-165.5) vs 108.2 (93.0-132.1) g, $\mathrm{p}=0.02)$. When indexed to BSA, the LVM remained significantly higher 
Table 1 Baseline characteristics of the patients with BAV and CTL

\begin{tabular}{llll}
\hline & CTL $(\mathbf{n}=85)$ & BAV $(\mathbf{n}=\mathbf{7 9})$ & P values \\
\hline Age $($ years $)$ & $28(24-31)$ & $38(29-51)$ & $<0.0001$ \\
\hline Men, $n(\%)$ & $37(44)$ & $52(66)$ & 0.004 \\
\hline Weight $(\mathrm{kg})$ & $67.1(57.5-80.6)$ & $73.1(64.3-84.0)$ & 0.046 \\
\hline Height $(\mathrm{m})$ & $1.70 \pm 0.09$ & $1.71 \pm 0.10$ & 0.48 \\
\hline BSA $\left(\mathrm{m}^{2}\right)$ & $1.81 \pm 0.22$ & $1.86 \pm 0.21$ & 0.13 \\
\hline BMI $\left(\mathrm{kg} / \mathrm{m}^{2}\right)$ & $23.5(21.3-26.4)$ & $25.4(22.8-27.7)$ & 0.01 \\
\hline NYHA class III or IV, $\mathrm{n}(\%)$ & $0(0)$ & $1(1)$ & - \\
\hline ICD, $\mathrm{n}(\%)$ & $0(0)$ & $0(0)$ & - \\
\hline Oral anticoagulation, $\mathrm{n}(\%)$ & $0(0)$ & $4(5)$ & - \\
\hline LVEDV $(\mathrm{mL})$ & $144.5(127.8-168.7)$ & $163.0(133.3-204.2)$ & 0.03 \\
\hline LVESV $(\mathrm{mL})$ & $47.4(38.4-56.3)$ & $53.0(40.7-80.7)$ & 0.03 \\
\hline LVEDVi $\left(\mathrm{mL} / \mathrm{m}^{2}\right)$ & $81.9(75.2-87.6)$ & $86.3(74.0-101.8)$ & 0.07 \\
\hline LVESVi $\left(\mathrm{mL} / \mathrm{m}^{2}\right)$ & $26.0(23.2-30.6)$ & $30.3(22.2-41.2)$ & 0.02 \\
\hline LVSV $(\mathrm{mL})$ & $99.5(86.6-111.4)$ & $108.0(86.8-123.0)$ & 0.12 \\
\hline LVSVi $\left(\mathrm{mL} / \mathrm{m}^{2}\right)$ & $55.3(50.6-60.1)$ & $55.8(49.6-65.0)$ & 0.50 \\
\hline LVCO $(\mathrm{L} / \mathrm{min})$ & $6.9(5.8-8.2)$ & $7.4(6.2-8.5)$ & 0.37 \\
\hline LVEF $(\%)$ & $68(64-71)$ & $65(59-72)$ & 0.08 \\
\hline LVM $(\mathrm{g})$ & $108.2(93.0-132.1)$ & $131.5(97.6-165.5)$ & 0.02 \\
\hline LVMi $\left(\mathrm{g} / \mathrm{m}^{2}\right)$ & $61.1(53.9-69.5)$ & $71.7(58.9-81.4)$ & 0.001 \\
\hline
\end{tabular}

Continuous variables are expressed as mean \pm SD or median (25th percentile-75th percentile). Categorical variables as $n$ (\%).

BAV, bicuspid aortic valve; BMI, body mass index; BSA, body surface area; CTL, control subjects; ICD, implanted cardioverter-defibrillator; LVCO, left ventricle cardiac output; LVEDV, left ventricle end-diastolic volume; LVEDVi, LVEDV indexed by BSA; LVEF, left ventricle ejection fraction; LVESV, left ventricle end-systolic volume; LVESVi, LVESV indexed by BSA; LVM, left ventricle mass; LVMi, LVM indexed by BSA; LVSV, left ventricle stroke volume;NYHA, New York Heart Association.

in patients with BAV than in CTL (71.7 (58.9-81.4) vs $\left.61.1(53.9-69.5) \mathrm{g} / \mathrm{m}^{2}, \mathrm{p}=0.001\right)$. LVEF was not different between CTL (68 (64-71)\%; range of LVEF: 49\%-83\%) and patients with BAV (65 (59-72)\%; range of LVEF: $35 \%-86 \%)(\mathrm{p}=0.08)$.

\section{Prevalence of LVNC criteria in patients with BAV versus CTL}

The prevalence of CTL and patients with BAV with NC/C ratios superior to 2.0, 2.3 and 2.5, assessed at end-systole and end-diastole, is presented in figure 2 . When we used the three cut-points described above for the NC/C ratios assessed in systole, no statistically significant difference was observed between CTL and patients with BAV for the prevalence of LVNC criteria (all $p>0.13$ ). When analyses of $\mathrm{NC} / \mathrm{C}$ ratios were performed in diastole using the three cut-points described above, LVNC criteria were met more often in CTL than in patients with BAV (all $\mathrm{p}<0.006$ ).

The prevalence of CTL and patients with BAV meeting the LVNC criteria is presented in figure 3A. Among the $85 \mathrm{CTL}$ and 79 patients with BAV, 15 CTL $(17.6 \%)$ vs 8 BAV $(10.1 \% ; \mathrm{p}=0.17)$ fulfilled Jenni $e t a l$ s criterion $(>2.0$ in systole); 69 CTL $(81.2 \%)$ vs 49 BAV $(62.0 \%$; $p=0.006)$ fulfilled Petersen $e t a l$ 's criterion ( $>2.3$ in diastole); 66 CTL $(77.6 \%)$ vs 43 BAV (54.4\%; $\mathrm{p}=0.002)$ fulfilled Fazio et als scriterion ( $>2.5$ in diastole); and 15 CTL $(17.6 \%)$ vs
7 BAV (8.9\%; $\mathrm{p}=0.10$ ) fulfilled all three criteria. Petersen et $a l$ and Fazio $e t a l$ s LVNC criteria were met more often by CTL ( $p=0.006$ and $p=0.002$, respectively) than patients with BAV. However, according to Jenni $e t a l$ 's LVNC criterion, the difference between patients with $\mathrm{BAV}$ and CTL was not statistically significant $(p=0.17)$. There was no statistical difference between patients with BAV and CTL meeting all criteria $(\mathrm{p}=0.10)$.

\section{Subanalyses in patients with BAV with and without valve dysfunction}

Among the 79 patients with BAV, 37 (46.8\%) had no significant valve dysfunction (group ND), 25 (31.7\%) had a predominant and significant (>mild) AS (group AS) and $17(21.5 \%)$ had a predominant and significant (>mild) AR (group AR). Baseline characteristics of these three BAV subgroups are presented in table 2 . The prevalence of patients with $\mathrm{NC} / \mathrm{C}$ ratios $>2.0,2.3$ and 2.5 at end-systole and end-diastole (online supplementary figure 1) as well as the prevalence of patients meeting the previously proposed criteria for LVNC (figure 3B) were not statistically different between these three BAV subgroups.

\section{Adjustment for the prevalence of LVNC criteria}

Logistic regression analyses were used to investigate the association between the aortic valve morphology (BAV vs 
A

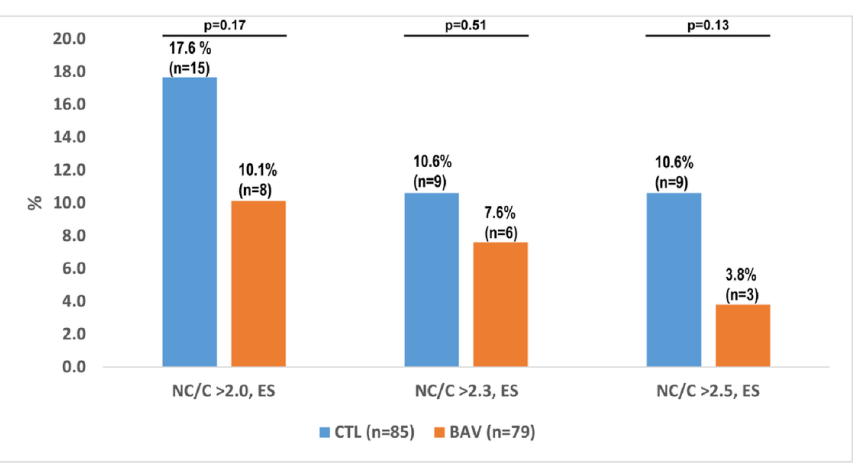

B

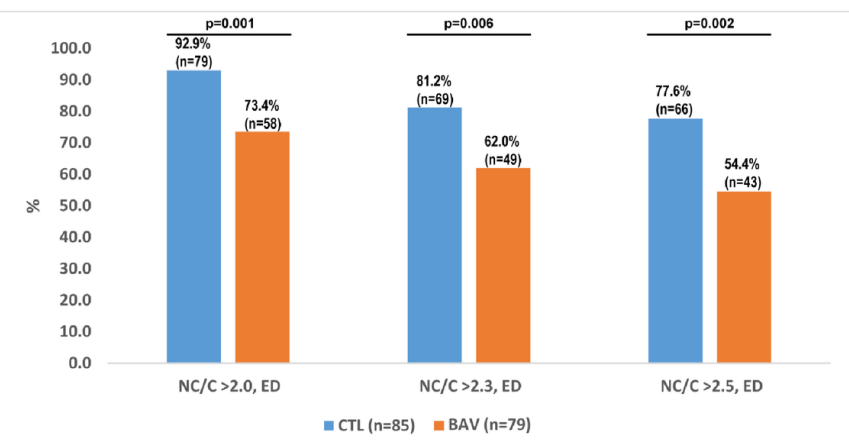

Figure 2 Prevalence of CTL and patients with BAV with a $\mathrm{NC} / \mathrm{C}$ ratio $>2.0,2.3$ and 2.5 at end-diastole and end-systole. (A) CTL and patients with BAV with a NC/C ratio superior to 2.0, 2.3 and 2.5 at end-systole. (B) CTL and patients with $\mathrm{BAV}$ with a NC/C ratio superior to 2.0, 2.3 and 2.5 at enddiastole. BAV, bicuspid aortic valve; CTL, control subjects; $\mathrm{ED}$, end-diastole; ES, end-systole; NC/C, non-compacted/ compacted myocardium ratio.

tricuspid aortic valve) and the presence of LVNC criteria according to the three methods. The results of the univariable and multivariable analyses are presented in table 3 .

In univariable analyses, BAV was associated with a lower likelihood of presenting LVNC according to Petersen et al $(\mathrm{OR}=0.38,95 \%$ CI 0.19 to $0.77 ; \mathrm{p}=0.007)$ and Fazio et al's $(\mathrm{OR}=0.34,95 \%$ CI 0.17 to $0.68 ; \mathrm{p}=0.002)$ LVNC criteria. In the multivariable model (model 1, table 3) adjusted for age and sex, BAV was significantly and inversely associated with the presence of Fazio et al's LVNC criterion $(\mathrm{OR}=0.40,95 \%$ CI 0.17 to $0.91 ; \mathrm{p}=0.03)$. With further adjustment for valve dysfunction (stenosis or regurgitation more than mild) (model 2, table 3), BAV remained associated with a lower prevalence of LVNC using Fazio et al's criterion $(\mathrm{OR}=0.33,95 \%$ CI 0.13 to $0.83 ; \mathrm{p}=0.02)$. Finally, with an adjustment for age, sex, valve dysfunction, indexed LVM, LVEDVi and LVEF (model 3, table 3), BAV still remained inversely associated with Jenni et al's criterion $(\mathrm{p}=0.04)$, Fazio et al's criterion $(\mathrm{p}=0.004)$ and all three criteria $(\mathrm{p}=0.04)$.

\section{DISCUSSION}

The main findings of the present study are: (1) patients with BAV do not meet LVNC criteria more often than
A
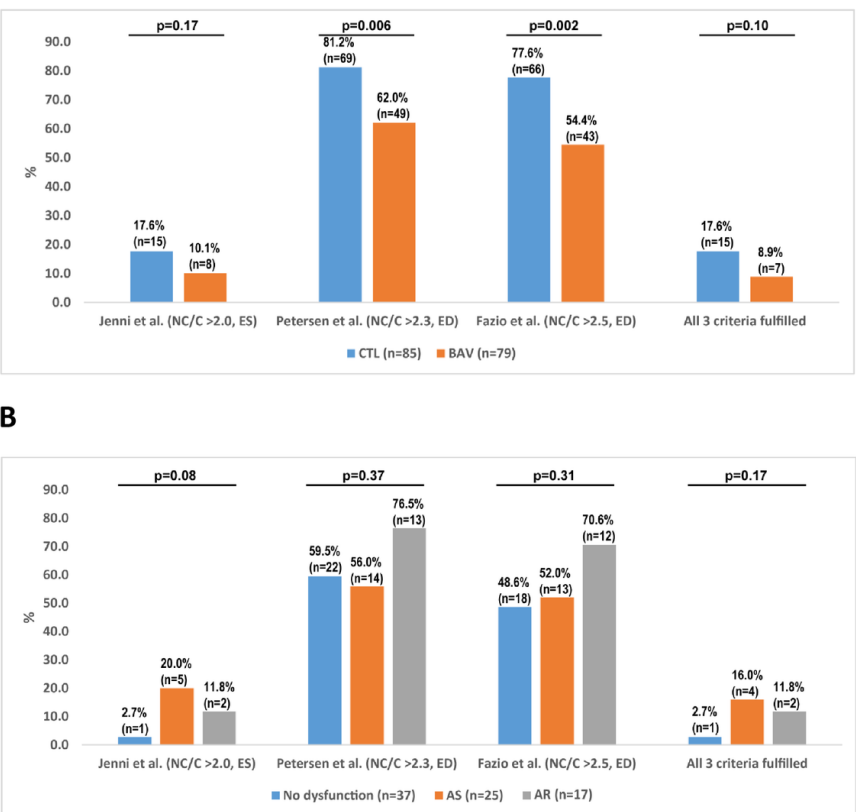

Figure 3 Prevalence of subjects meeting the left ventricle non-compaction (LVNC) criteria. (A) Prevalence of CTL and patients with BAV meeting the LVNC criteria of Jenni et al (NC/C ratio $>2.0, E S)$, Petersen et al (NC/C ratio $>2.3$, ED), Fazio et al (NC/C ratio $>2.5$, ED) and all three criteria. (B) Prevalence of patients with BAV meeting the LVNC criteria of Jenni et al (NC/C ratio $>2.0$, ES), Petersen et al (NC/C ratio $>2.3$, ED), Fazio et al (NC/C ratio $>2.5$, ED) and all three criteria, according to the presence of valve dysfunction. $\mathrm{AR}$, aortic regurgitation; AS, aortic stenosis; BAV, bicuspid aortic valve; CTL, control subjects; ED, end-diastole; ES, end-systole; NC/C, non-compacted/compacted myocardium ratio.

healthy CTL, according to the $\mathrm{NC} / \mathrm{C}$ ratio thresholds of three methods previously published in the literature; (2) there is no difference in the prevalence of LVNC criteria among patients with BAV with no aortic valve dysfunction, significant AS and significant AR; and (3) in multivariable analyses including potential confounders, BAV morphology was not associated with increased prevalence of LVNG criteria.

\section{LVNC in the general population}

LVNC is a rare cardiomyopathy. However, several studies showed that even in subjects without cardiovascular disease, a significant proportion met the current LVNC criteria. Forty-three per cent $(43 \%)$ of subjects without cardiac disease or hypertension fulfilled the criterion of Petersen et al in the Multi-Ethnic Study of Atherosclerosis $^{28}$ and $46.2 \%$ healthy volunteers in another study by André et $a l^{29}{ }^{29}$ Likewise, Weir-McCall et $a l^{30}$ showed that $74 \%$ of subjects free of any cardiovascular disease fulfilled at least one of the four CMR diagnostic criteria they underwent in the study, with $62.8 \%$ meeting Petersen et al's criterion. These studies underline the poor specificity of the current diagnostic criteria for LVNC, and this lack of specificity results in overdiagnosis in normal 
Table 2 Characteristics of subgroups of patients with BAV

\begin{tabular}{|c|c|c|c|c|}
\hline & $\begin{array}{l}\text { BAV with no aortic } \\
\text { valve dysfunction } \\
(n=37)\end{array}$ & $\begin{array}{l}\text { BAV with AS } \\
(n=25)\end{array}$ & $\begin{array}{l}\text { BAV with AR } \\
(n=17)\end{array}$ & $P$ values \\
\hline Men, n (\%) & $25(68)$ & $13(52)$ & $14(82)$ & 0.12 \\
\hline Weight (kg) & $76(67-89)$ & $72(59-77)$ & $68(65-95)$ & 0.37 \\
\hline $\mathrm{BMI}\left(\mathrm{kg} / \mathrm{m}^{2}\right)$ & $25.8(23.8-28.3)$ & $25.3(23.2-26.9)$ & $22.9(21.7-29.1)$ & 0.67 \\
\hline NYHA class III or IV, n (\%) & $1(3)$ & $0(0)$ & $0(0)$ & 0.52 \\
\hline ICD, n (\%) & $0(0)$ & $0(0)$ & $0(0)$ & - \\
\hline Oral anticoagulation, $\mathrm{n}(\%)$ & $3(8)$ & $0(0)$ & $1(6)$ & 0.36 \\
\hline LVESVi (mL/m²) & $32.1(27.6-39.8)^{\star}$ & $22.4(15.8-27.7)^{*} \dagger$ & $44.2(31.0-52.7) \dagger$ & 0.0001 \\
\hline LVSV (mL) & 108.5 (83.8-121.9)‡ & $98.8(82.3-116.5) \dagger$ & $133.1(97.2-151.9) \dagger \ddagger$ & 0.01 \\
\hline LVSVi $\left(\mathrm{mL} / \mathrm{m}^{2}\right)$ & 54.7 (48.0-62.3)‡ & $54.6(46.5-63.1) \dagger$ & $68.4(54.6-75.5) \dagger \ddagger$ & 0.006 \\
\hline LVCO (L/min) & $7.0(5.7-8.3) \ddagger$ & $6.9(6.0-7.8) \dagger$ & $8.8(7.5-9.8) \dagger \ddagger$ & 0.002 \\
\hline $\operatorname{LVEF}(\%)$ & $61(57-69)^{\star}$ & $71(67-75)^{*} \dagger$ & $62(55-66) \dagger$ & 0.0001 \\
\hline LVM (g) & $134.1(96.8-157.8) \ddagger$ & $113.2(92.5-153.1) \dagger$ & 165.5 (127.8-187.5)†† & 0.03 \\
\hline LVMi $\left(g / m^{2}\right)$ & 71.0 (58.3-79.5)† & $63.4(58.1-77.9) \dagger$ & $78.1(72.1-94.2) \dagger \ddagger$ & 0.03 \\
\hline
\end{tabular}

${ }^{*} \mathrm{P}<0.05$ between $\mathrm{BAV}$ with no aortic valve dysfunction and BAV with AS.

$\dagger P<0.05$ between BAV with AS and BAV with AR.

$\ddagger \mathrm{P}<0.05$ between BAV with no aortic valve dysfunction and BAV with AR.

$\mathrm{AR}$, aortic regurgitation; $\mathrm{AS}$, aortic stenosis;BAV, bicuspid aortic valve;BMI, body mass index;BSA, body surface area;ICD, implanted cardioverter-defibrillator;LVCO, left ventricle cardiac output;LVEDV, left ventricle end-diastolic volume;LVEDVi, LVEDV indexed by BSA;LVEF, left ventricle ejection fraction;LVESV, left ventricle end-systolic volume;LVESVi, LVESV indexed by BSA;LVM, left ventricle mass;LVMi, LVM indexed by BSA;LVSV, left ventricle stroke volume;LVSVi, LVSV indexed by BSA;NYHA, New York Heart Association.

individuals. ${ }^{30}$ Furthermore, there is a poor agreement between studies and between observers. ${ }^{31} 32$

\section{BAV and LVNC}

LVNC is a very rare entity, thus it is difficult to assess its exact prevalence and it explains the different and variable values reported in the literature. The objective of the present study was not to determine the actual prevalence of LVNC in the patients with BAV or in the CTL group but rather to determine if the prevalence of patients with BAV meeting the LVNC criteria was higher than that in healthy CTL, as suggested by some studies. ${ }^{1920}$ The hypothesis that BAV subjects may present more LVNC than the general population is legitimate as both anomalies may be related to the Notch pathway. ${ }^{33} 34$ In line with this hypothesis, some studies suggested that the prevalence of LVNC as assessed by echocardiography is higher in BAV subjects than in the general population. ${ }^{19}{ }^{20}$ However, these previous studies did not include a CTL group. The present study has the advantage of comparing patients with BAV with a control group of healthy CTL, which, to our knowledge, is the first study to do so. The difference in age between BAV and CTL can be explained by the fact that CTL were recruited in a study that required an age between 18 and 35 years old as one of the inclusion criteria. The difference in male sex prevalence between the two groups can also be reasonably explained by the fact that BAV is more prevalent in men than in women. ${ }^{1535}$ BAV subjects had higher LV volumes and mass than CTL subjects potentially because there are more men in BAV group but also because some of the patients with BAV had significant aortic valve dysfunction (such as AR). According to the present study, there is no evidence that LVNC is more prevalent in BAV subjects than in the general population. Even when we adjusted for the differences between BAV and CTL, LVNC was not more frequent in BAV than in CTL. The multivariable analyses even revealed lower prevalence of LVNC criteria in the BAV versus the CTL. The potential factors that may have contributed to the lower prevalence of LVNC in the CTL subjects include: (1) a potentially better visualisation and delineation of the trabeculations in the CTL; (2) the younger age of the CTL subjects; and (3) the larger LV pressure and volume overload related to aortic valve dysfunction in the patients with BAV. 
Limitations of the current methods used for identification of LVNC

Echocardiography and CMR are the most common imaging modalities used to diagnose LVNC. Several diagnostic methods and criteria have been described over the years, however, there is still no consensus about which method to adopt for each modality. Furthermore, the diagnostic parameters and criteria proposed in the literature are based on studies including relatively small sample size. ${ }^{1} 26273637$ LVNC is generally identified using the $\mathrm{NC} / \mathrm{C}$ ratio and the thresholds that have been previously published. Jenni et al used a $\mathrm{NC} / \mathrm{C}$ ratio $>2.0$ in systole by echocardiography to define the presence of LVNC. ${ }^{1}$ This is the most frequently used method in clinical practice as echocardiography is the primary cardiac imaging modality. We used this method for our study, although it has not been previously validated for CMR. However, when using this method for both patients with BAV and CTL, no difference was found with regard to the prevalence of LVNC. Petersen $e t a l^{26}$ proposed a NC/C ratio $>2.3$ assessed by CMR in diastole to define LVNC, whereas Fazio $e t a l^{27}$ used a NC/C ratio $>2.5$ in diastole by CMR. In our study, these criteria of Petersen et al and Fazio et al were met by a large number of patients with BAV and even more by CTL. These findings as well as those of previous studies suggest that these criteria may be too sensitive. ${ }^{24}{ }^{29}{ }^{30}$ Further studies in large cohorts of patients are necessary to re-evaluate and eventually revise the criteria of LVNC especially for CMR.

Whether LVNC should be assessed in systole or in diastole is also still a matter of debate. ${ }^{431} 38$ While most of the current diagnostic criteria are assessed in diastole, Stacey et $a \hat{l}^{39}$ are in favour of an assessment of $\mathrm{NC} / \mathrm{C}$ ratio in systole as they reported that systolic criteria are associated with higher rate of events and lower LVEF and myocardial thickening; whereas with diastolic criteria, there was a trend for overdiagnosis of LVNC. Applying the three cut-off values of NC/C ratios (from Jenni et al, Petersen $e t$ $a l$ and Fazio $e t a l$ ) to the end-diastolic values obviously led to an overdiagnosis of LVNC in the present study.

\section{Clinical implications}

Subjects of BAV are at increased risk of developing severe aortic valve dysfunction and/or aortopathy. These subjects thus require a life-long follow-up by Doppler echocardiography and/or CT. The results of this study suggest that subjects with BAV do not present more LVNC/hypertrabeculation than the healthy subjects free of cardiovascular disease or congenital anomaly. Hence, there is no evidence that BAV subjects may be at higher risk of developing LVNC cardiomyopathy compared with the general population. These results are reassuring and suggest that systematic screening for LVNC by CMR is not necessary.

Moreover, current echocardiographic and CMR methods and criteria obviously lead to major overdiagnosis of LVNC. There is no gold standard method to assess LVNC apart from ex vivo tissue analyses, nevertheless, 
CMR may be the most accurate and reproducible imaging modality to assess LVNC as it has a high spatial resolution, therefore allowing a good delineation between the trabeculated and compacted layers. However, further studies in larger number of subjects are necessary to adjust and validate the $\mathrm{NC} / \mathrm{C}$ ratios to accurately diagnose clinically significant LVNC and studies focusing on genetic susceptibility to LVNC may also play a crucial role.

\section{Study limitations}

The main limitations of the study are the retrospective design and the presence of differences in the baseline characteristics between BAV and healthy CTL. However, even after adjusting for these differences, BAV did not appear to be associated with higher likelihood of LVNC. In addition, the subjects with BAV included patients with valve dysfunction. However, the presence of dysfunction, stenosis or regurgitation did not significantly influence the distribution of LVNC among patients with BAV. Furthermore, one may argue that our study could present a type II error and not detect a potential existing difference between patients with BAV and healthy CTL. However, while applying the exact same methods to assess LVNC criteria in both patients with BAV and CTL, the results persisted all along the analyses, showing that patients with BAV do not present more LVNC criteria than CTL. Our study population consisted exclusively of Caucasian (French Canadian), so we could not investigate LVNC according to races/ethnicity. Finally, as only one observer did the analyses for LVNC, we could not evaluate the interobserver measurement variability.

\section{CONCLUSION}

In this study, the proportion of patients with BAV fulfilling the criteria for LVNC was not higher than that of healthy CTL. Therefore, this study refutes the hypothesis that patients with BAV have more LVNC than the general population and are at higher risk for development of LVNC cardiomyopathy. These findings do not support systematic screening for LVNC in subjects with BAV.

Acknowledgements We thank Isabelle Fortin, Jocelyn Beauchemin, Céline Boutin, Louise Marois, Martine Poulin, Martine Parent, Michèle Cyr and Karine Bibeau for their help in data collection and management.

Contributors MS: study management, acquisition, analyses and interpretation of collected data, preparation of the manuscript. RC, LT: participation in the study management, acquisition of data and critical revisions of the manuscript. EG: CMR measurements, critical revisions of the manuscript. MAC, ES, PC, HTM, FLV: critical revisions of the manuscript. EB, MA: coinvestigators of the PROGRESSA study and critical revisions of the manuscript. PP: principal investigator of the PROGRESSA study, supervision of data collection, analyses and interpretation and critical revisions of the manuscript. EL: coinvestigator of the PROGRESSA study, hypothesis and methods refinement, design and supervision of the CMR imaging data collection/analysis and critical revision of the manuscript.

Funding This work was supported by grants MOP-114997, MOP-2455048 and FDN-143225 from Canadian Institutes of Health Research (ClHR), Ottawa, Ontario, Canada, and a grant from the Foundation of the Quebec Heart and Lung Institute. $\mathrm{RC}$ is supported by a postdoctoral fellowship grant from CIHR. MA is a Research Scholar from the Fonds de Recherche Québec-Santé (FRQS), Montreal, Québec, Canada. PP holds the Canada Research Chair in Valvular Heart Diseases from CIHR,
Ottawa, Ontario, Canada. EL is a Senior Clinical Research Scholar from the Fonds de Recherche Québec-Santé (FRQS), Montreal, Québec, Canada.

Competing interests The authors have reported no relationships relevant to the content of this paper to disclose.

Patient consent Not required.

Ethics approval Ethics Committee of the Quebec Heart and Lung Institute.

Provenance and peer review Not commissioned; externally peer reviewed.

Data statement № additional unpublished data are available to be shared.

Open access This is an open access article distributed in accordance with the Creative Commons Attribution Non Commercial (CC BY-NC 4.0) license, which permits others to distribute, remix, adapt, build upon this work non-commercially, and license their derivative works on different terms, provided the original work is properly cited, appropriate credit is given, any changes made indicated, and the use is non-commercial. See: http://creativecommons.org/licenses/by-nc/4.0/

\section{REFERENCES}

1. Jenni R, Oechslin E, Schneider J, et al. Echocardiographic and pathoanatomical characteristics of isolated left ventricular non-compaction: a step towards classification as a distinct cardiomyopathy. Heart 2001;86:666-71.

2. Oechslin E, Jenni R. Left ventricular non-compaction revisited: a distinct phenotype with genetic heterogeneity? Eur Heart $J$ 2011;32:1446-56.

3. Hussein A, Karimianpour A, Collier P, et al. Isolated noncompaction of the left ventricle in adults. J Am Coll Cardiol 2015;66:578-85.

4. Finsterer J, Stöllberger C, Towbin JA. Left ventricular noncompaction cardiomyopathy: cardiac, neuromuscular, and genetic factors. Nat Rev Cardiol 2017;14:224-37.

5. Arbustini E, Weidemann F, Hall JL. Left ventricular noncompaction: a distinct cardiomyopathy or a trait shared by different cardiac diseases? J Am Coll Cardiol 2014;64:1840-50.

6. Ritter M, Oechslin E, Sütsch G, et al. Isolated noncompaction of the myocardium in adults. Mayo Clin Proc 1997;72:26-31.

7. Aras D, Tufekcioglu O, Ergun K, et al. Clinical features of isolated ventricular noncompaction in adults long-term clinical course, echocardiographic properties, and predictors of left ventricular failure. J Card Fail 2006;12:726-33.

8. Sandhu R, Finkelhor RS, Gunawardena DR, et al. Prevalence and characteristics of left ventricular noncompaction in a community hospital cohort of patients with systolic dysfunction. Echocardiography 2008;25:8-12.

9. Lilje C, Rázek V, Joyce JJ, et al. Complications of non-compaction of the left ventricular myocardium in a paediatric population: a prospective study. Eur Heart J 2006;27:1855-60.

10. Towbin JA, Lorts A, Jefferies JL. Left ventricular non-compaction cardiomyopathy. Lancet 2015;386:813-25.

11. Elliott $P$, Andersson $B$, Arbustini $E$, et al. Classification of the cardiomyopathies: a position statement from the European Society Of Cardiology Working Group on Myocardial and Pericardial Diseases. Eur Heart J 2008;29:270-6.

12. Maron BJ, Towbin JA, Thiene G, et al. Contemporary definitions and classification of the cardiomyopathies: an American Heart Association Scientific Statement from the Council on Clinical Cardiology, Heart Failure and Transplantation Committee; Quality of Care and Outcomes Research and Functional Genomics and Translational Biology Interdisciplinary Working Groups; and Council on Epidemiology and Prevention. Circulation 2006;113:1807-16.

13. Richardson P, McKenna W, Bristow M, et al. Report of the 1995 World Health Organization/International Society and Federation of Cardiology Task Force on the Definition and Classification of cardiomyopathies. Circulation 1996;93:841-2.

14. Roberts WC. The congenitally bicuspid aortic valve. A study of 85 autopsy cases. Am J Cardiol 1970;26:72-83.

15. Basso C, Boschello M, Perrone C, et al. An echocardiographic survey of primary school children for bicuspid aortic valve. Am J Cardiol 2004;93:661-3.

16. Fedak PW, Verma S, David TE, et al. Clinical and pathophysiological implications of a bicuspid aortic valve. Circulation 2002;106:900-4.

17. Ward C. Clinical significance of the bicuspid aortic valve. Heart 2000;83:81-5.

18. Aboulhosn J, Child JS. Left ventricular outflow obstruction: subaortic stenosis, bicuspid aortic valve, supravalvar aortic stenosis, and coarctation of the aorta. Circulation 2006;114:2412-22. 
19. Agarwal A, Khandheria BK, Paterick TE, et al. Left ventricular noncompaction in patients with bicuspid aortic valve. J Am Soc Echocardiogr 2013;26:1306-13.

20. Stähli BE, Gebhard C, Biaggi P, et al. Left ventricular noncompaction: prevalence in congenital heart disease. Int $J$ Cardiol 2013;167:2477-81.

21. Thiele H, Nagel E, Paetsch I, et al. Functional cardiac MR imaging with steady-state free precession (SSFP) significantly improves endocardial border delineation without contrast agents. J Magn Reson Imaging 2001;14:362-7.

22. Hendel RC, Patel MR, Kramer CM, et al. ACCF/ACR/SCCT/SCMR/ ASNC/NASCI/SCAI/SIR 2006 appropriateness criteria for cardiac computed tomography and cardiac magnetic resonance imaging: a report of the American College of Cardiology Foundation Quality Strategic Directions Committee Appropriateness Criteria Working Group, American College of Radiology, Society of Cardiovascular Computed Tomography, Society for Cardiovascular Magnetic Resonance, American Society of Nuclear Cardiology, North American Society for Cardiac Imaging, Society for Cardiovascular Angiography and Interventions, and Society of Interventional Radiology. J Am Coll Cardiol 2006;48:1475-97.

23. Le Ven F, Bibeau K, De Larochellière É, et al. Cardiac morphology and function reference values derived from a large subset of healthy young Caucasian adults by magnetic resonance imaging. Eur Heart J Cardiovasc Imaging 2016;17:981-90.

24. Tizón-Marcos $\mathrm{H}$, de la Paz Ricapito M, Pibarot P, et al. Characteristics of trabeculated myocardium burden in young and apparently healthy adults. Am J Cardiol 2014;114:1094-9.

25. Cerqueira MD, Weissman NJ, Dilsizian V, et al. Standardized myocardial segmentation and nomenclature for tomographic imaging of the heart. A statement for healthcare professionals from the Cardiac Imaging Committee of the Council on Clinical Cardiology of the American Heart Association. Circulation 2002;105:539-42.

26. Petersen SE, Selvanayagam JB, Wiesmann F, et al. Left ventricular non-compaction: insights from cardiovascular magnetic resonance imaging. J Am Coll Cardiol 2005;46:101-5.

27. Fazio G, Novo G, D'Angelo L, et al. Magnetic resonance in isolated noncompaction of the ventricular myocardium. Int $J$ Cardiol 2010;140:367-9.
28. Kawel N, Nacif M, Arai AE, et al. Trabeculated (noncompacted) and compact myocardium in adults: the multi-ethnic study of atherosclerosis. Circ Cardiovasc Imaging 2012;5:357-66.

29. André F, Burger A, Loßnitzer D, et al. Reference values for left and right ventricular trabeculation and non-compacted myocardium. Int J Cardiol 2015;185:240-7.

30. Weir-McCall JR, Yeap PM, Papagiorcopulo C, et al. Left ventricular noncompaction: anatomical phenotype or distinct cardiomyopathy? J Am Coll Cardiol 2016;68:2157-65.

31. Kohli SK, Pantazis AA, Shah JS, et al. Diagnosis of left-ventricular non-compaction in patients with left-ventricular systolic dysfunction: time for a reappraisal of diagnostic criteria? Eur Heart J 2008;29:89-95.

32. Saleeb SF, Margossian R, Spencer CT, et al. Reproducibility of echocardiographic diagnosis of left ventricular noncompaction. $J$ Am Soc Echocardiogr 2012;25:194-202.

33. de la Pompa JL, Epstein JA. Coordinating tissue interactions: notch signaling in cardiac development and disease. Dev Cell 2012;22:244-54.

34. Luxán G, D'Amato G, MacGrogan D, et al. Endocardial notch signaling in cardiac development and disease. Circ Res 2016;118:e1-e18.

35. Tutar E, Ekici F, Atalay S, et al. The prevalence of bicuspid aortic valve in newborns by echocardiographic screening. Am Heart $J$ 2005;150:513-5.

36. Jacquier A, Thuny F, Jop B, et al. Measurement of trabeculated left ventricular mass using cardiac magnetic resonance imaging in the diagnosis of left ventricular non-compaction. Eur Heart $J$ 2010;31:1098-104.

37. Chin TK, Perloff JK, Williams RG, et al. Isolated noncompaction of left ventricular myocardium. A study of eight cases. Circulation 1990;82:507-13

38. Gati S, Rajani R, Carr-White GS, et al. Adult left ventricular noncompaction: reappraisal of current diagnostic imaging modalities. JACC Cardiovasc Imaging 2014;7:1266-75.

39. Stacey RB, Andersen MM, St Clair M, et al. Comparison of systolic and diastolic criteria for isolated LV noncompaction in CMR. JACC Cardiovasc Imaging 2013;6:931-40. 